\title{
QRS duration: a simple marker for predicting cardiac mortality in ICD patients with heart failure
}

\author{
L Bode-Schnurbus, D Böcker, M Block, R Gradaus, A Heinecke, G Breithardt, \\ M Borggrefe
}

See end of article for authors' affiliations

Correspondence to:

Dr Martin Borggrefe,

Department of Internal

Medicine, Medical Faculty

of the University of

Heidelberg in Mannheim,

Theodor Kutzer Ufer 1-3,

D-68135 Mannheim,

Germany;

martin.borggrefe@

med.ma.uni-heidelberg.de

Accepted 7 April 2003
Background: Patients resuscitated from ventricular tachyarrhythmias benefit from implantable cardioverter-defibrillators (ICDs) as opposed to medical treatment. Patients with increased QRS duration receiving an ICD in the presence of heart failure are at greatest risk of cardiac death and benefit most from ICD therapy.

Objective: To determine whether an increased QRS duration predicts cardiac mortality in ICD recipients.

Design: Consecutive patients with heart failure in New York Heart Association functional class III were grouped according to QRS duration ( $150 \mathrm{~ms}, \mathrm{n}=139$, group $1 ; v \geqslant 150 \mathrm{~ms}, \mathrm{n}=26$, group 2 ) and followed up for (mean (SD)) 23 (20) months.

Patients: 165 patients were studied ( $80 \%$ men, $20 \%$ women); $73 \%$ had coronary artery disease and $18 \%$ had dilated cardiomyopathy. Their mean age was 62 (10) years and mean ejection fraction (EF) was $33(14) \%$. They presented either with ventricular tachycardia (VT) or ventricular fibrillation (VF).

Main outcome measures: Overall and cardiac mortality; recurrence rates of VT, fast VT, or VF. Results: Mean left ventricular EF did not differ between group 1 (33 (13)\%) and group 2 (31 (15)\%). Forty patients died (34 cardiac deaths). There was no difference in survival between patients with $\mathrm{EF}>35 \%$ and $\leqslant 35 \%$. Cardiac mortality was significantly higher in group 2 than in group $1131.3 \%$ at 12 months and $46.6 \%$ at 24 months, $v 9.5 \%$ at 12 months and $18.2 \%$ at 24 months, respectively; $\mathrm{p}=0.04)$. The recurrence rate of VT was similar in both groups.

Conclusions: Within subgroups at highest risk of cardiac death, QRS duration - a simple non-invasive index-predicts outcome in ICD recipients in the presence of heart failure.
$\mathrm{H}$ eart failure is a significant public health concern worldwide and it is estimated that $1-2$ million people in the USA have congestive heart failure. The annual incidence of new cases is approximately 400000 a year. ${ }^{12}$ Patients with congestive heart failure have a poor prognosis and many studies have shown mortalities of around $15 \%$ in the first year and $30 \%$ in the second year. ${ }^{3}$

Although significant progress has been made in the treatment of heart failure, patients continue to have a poor quality of life and an unacceptably high mortality. Prediction of death in patients with congestive heart failure is challenging. The clinical events which predict the highest subsequent risk are also the most serious presenting events-namely, symptomatic ventricular tachyarrhythmias (including cardiac arrest) and syncope. Among those patients surviving such an event, the risk of recurrent ventricular tachycardia (VT) is $>30 \%$ at $1-3$ years, while the actual risk of subsequent sudden death for patients presenting with syncope is $45 \%$ at one year. ${ }^{4}$

Three recent randomised clinical trials have provided evidence of increased survival in patients with resuscitated VT or ventricular fibrillation (VF) who received an implantable cardioverter defibrillator (ICD) compared with those who were treated with amiodarone. ${ }^{5-7}$ The overall benefit in survival, however, was small. Considering the high costs of ICD treatment, selection of patients most likely to benefit would make an ICD more cost effective.

Up to $50 \%$ of heart failure patients have intraventricular conduction delays that result in abnormal electrical depolarisation of the heart and mechanical asynchrony of the ventricles. ${ }^{8-14}$ Data from five studies that examined the relation between QRS duration and prognosis suggest that a wide QRS is an independent or contributing risk factor in patients with heart failure. ${ }^{10-14}$ Given the high costs and invasiveness of ICD implantation, it is important to determine which patients are likely to benefit most from treatment with these devices. For this reason, simple clinical indices have recently been introduced to identify patients at highest risk of death and benefiting most from ICD treatment, based on older age, poor ventricular function, and poor functional status. ${ }^{15} 16$

We hypothesised that within a group of patients with heart failure (New York Heart Association (NYHA) functional class III), the QRS duration may be an important independent predictor of cardiac death in ICD recipients. We therefore initiated a prospective follow up of 165 consecutive patients in NYHA grade III at the time of ICD implantation, and presenting with either VF or VT, and analysed the data obtained to determine whether an increased QRS duration predicted cardiac mortality.

\section{METHODS}

\section{Patient selection}

In a prospectively collected database, 603 consecutive patients who received an ICD with extended memory functions (intracardiac electrogram and RR interval storage) in combination with a transvenous endocardial lead system had a history of having survived either sudden cardiac death (cardiac

Abbreviations: AVID, antiarrhythmics versus implantable defibrillator trial; CAST, cardiac arrhythmia suppression trial; CIDS, Canadian implantable defibrillator study; EF, ejection fraction; GUSTO-I, global utilisation of streptokinase and t-PA for occluded coronary arteries; ICD, implantable cardioverter-defibrillator; MADIT, multicenter automatic defibrillator implantation trial; VF, ventricular fibrillation; VT, ventricular tachycardia 
Table 1 Patient characteristics

\begin{tabular}{|c|c|c|c|}
\hline & $\begin{array}{l}\text { QRS }<150 \mathrm{~ms} \\
(\mathrm{n}=139)\end{array}$ & $\begin{array}{l}\text { QRS } \geqslant 150 \mathrm{~ms} \\
(\mathrm{n}=26)\end{array}$ & $\begin{array}{l}\text { All patients } \\
(n=165)\end{array}$ \\
\hline Male/female (\%) & $80.6 / 19.4$ & $76.9 / 23.1$ & $80.0 / 20.0$ \\
\hline Age (years) (mean (SD)) & $61.8(9.7)$ & $61.7(9.9)$ & $61.8(9.7)$ \\
\hline Age range (years) & $14-82$ & $31-79$ & $14-82$ \\
\hline $\mathrm{EF}(\%)$ (mean (SD)) & $32.9(13.3)$ & $30.6(15.0)$ & $32.5(13.6)$ \\
\hline EF range $(\%)$ & 7 to 83 & 10 to 61 & 7 to 83 \\
\hline \multicolumn{4}{|l|}{ Underlying disease (n) } \\
\hline$C A D$ & $104(74.8 \%)$ & $16(61.5 \%)$ & $120(72.7 \%)$ \\
\hline DCM & $24(17.3 \%)$ & $5(19.2 \%)$ & $29(17.6 \%)$ \\
\hline ARVCM & $3(2.2 \%)$ & $1(3.8 \%)$ & $4(2.4 \%)$ \\
\hline Valvar & $5(3.6 \%)$ & $4(15.4 \%)$ & $9(5.5 \%)$ \\
\hline Other & $3(2.2 \%)$ & - & $3(1.8 \%)$ \\
\hline \multicolumn{4}{|l|}{ Presenting arrhythmia (n) } \\
\hline CA & $86(61.7 \%)$ & $18(69.1 \%)$ & $104(63.0 \%)$ \\
\hline Only VT & $51(36.7 \%)$ & $9(34.6 \%)$ & $60(36.4 \%)$ \\
\hline Only VF & $50(36.0 \%)$ & $8(30.8 \%)$ & $58(35.2 \%)$ \\
\hline $\mathrm{VT}+\mathrm{VF}$ & $29(20.9 \%)$ & $8(30.8 \%)$ & $37(22.4 \%)$ \\
\hline Others & $9(6.5 \%)$ & $1(3.8 \%)$ & $10(6.1)$ \\
\hline \multicolumn{4}{|l|}{ Drug treatment $(n)$} \\
\hline Class I AA drugs & $2(1,5 \%)$ & - & $2(1.3 \%)$ \\
\hline Class III AA drugs & $30(22.6 \%)$ & $8(33.3 \%)$ & $38(24.2 \%)$ \\
\hline$\beta$ Blocker & $38(28.4 \%)$ & $3(12.5 \%)$ & $41(25.9 \%)$ \\
\hline ACE inhibitor & $94(70.1 \%)$ & $21(87.5 \%)$ & $115(72.8 \%)$ \\
\hline Diuretic & $89(66.4 \%)$ & $19(79.2 \%)$ & $108(68.4 \%)$ \\
\hline Digitalis & $102(76.1 \%)$ & $20(83.3 \%)$ & $122(77.2 \%)$ \\
\hline \multicolumn{4}{|l|}{ Lead system (n) } \\
\hline Transvenous & $84(60.4 \%)$ & $18(69.2 \%)$ & $102(61.8 \%)$ \\
\hline Transvenous-subcutaneous & $53(38.1 \%)$ & $8(30.8 \%)$ & $61(37.0 \%)$ \\
\hline Epicardial & $2(1.4 \%)$ & - & $2(1.2 \%)$ \\
\hline
\end{tabular}

$A A$, antiarrhythmic; $A C E$, angiotensin converting enzyme; $A R V C M$, arrhythmogenic right ventricular cardiomyopathy; $C A$, cardiac arrest; $C A D$, coronary artery disease; $D C M$, dilated cardiomyopathy; $E F$, ejection fraction; VF, ventricular fibrillation; VT, ventricular tachycardia.

arrest) or sustained VT or syncope. All these patients gave written informed consent before device implantation.

From this database, 165 patients were identified who were in NYHA functional class III at the time of implantation. These patients formed the basis for further analysis. In all but two patients, a transvenous lead system was successfully implanted, in part in combination with a subcutaneous patch or an array electrode to achieve a sufficient defibrillation threshold. All implanted devices had the capability of either storing RR intervals or storing intracardiac electrograms. All patients had antitachycardia pacing capabilities in combination with cardioversion/defibrillation treatment features. Shock therapy was programmed to be non-committed.

\section{Follow up}

The follow up period of all patients started at the time of implantation, and patients were seen regularly on an outpatient basis at 2-3 month intervals. At each visit, the device was functionally tested (sensing, pacing impedance, and threshold) and interrogated for treated episodes. Primary end points were perioperative mortality (defined as death from any cause within the first 30 days following implantation), cardiac mortality, and sudden death mortality (defined as death within one hour of the onset of symptoms or unwitnessed death in a formerly stable patient). Recurrence of VT or fast VT ( $\geqslant 240$ beats/min) only, as suggested by Böcker and colleagues, was also determined. ${ }^{16}$ All treated episodes were classified by two independent cardiologists as inappropriate or appropriate, using the stored data.

\section{Statistical analysis}

QRS duration was measured in a 12 lead ECG taken at the time of implantation, using leads V3-V6. A mean of at least three complexes was used for further analysis. Univariate analyses were undertaken to detect differences between subgroups. Kaplan-Meier analysis was done using the end points all cause death, cardiac death, sudden death, VT recurrence at any rate, and recurrence of fast VT ( $\geqslant 240$ beats/min), to generate survival tables for each subgroup including the Breslow test. Patients were divided into two groups according to QRS duration. A cut off point of $150 \mathrm{~ms}$ was chosen, as it has recently been suggested that this identifies patients with heart failure who benefit from a biventricular pacing system. ${ }^{17}$

\section{RESULTS}

\section{Baseline characteristics of study groups}

In all, 165 patients in NYHA functional class III received a transvenous endocardial ICD system. In 61 patients this was combined with a subcutaneous array or a finger electrode. Only two patients underwent thoracotomy for implantation of an epicardial lead system. There were 132 men (80\%) and 33 women, mean (SD) age, 62 (10) years (table 1). The mean ejection fraction (EF) as assessed by left ventricular angiography was $33(14) \%$. A history of cardiac arrest was present in $63 \%$ of patients; $73 \%$ had coronary artery disease and $18 \%$ had dilated cardiomyopathy. At the time of implantation, 139 patients $(85 \%)$ had a QRS duration of $<150 \mathrm{~ms}$, while 26 (16\%) had a QRS duration of $150 \mathrm{~ms}$ or more. There were no significant differences in the clinical characteristics of these two groups. In addition, there were no differences in the use of either class III antiarrhythmic drugs or $\beta$ blockers.

The following devices were used: Guidant/CPI, either P2 or $\mathrm{P} 3$ in 28 patients; $\mathrm{PRx}$ in 14; $\mathrm{PRx} 2 / 3$ in 38 ; Mini in 13; Mini II in four; Medtronic 7216A and 7217B in 20; 7218 in seven; 7219 in 30; Jewel plus in five; Ventritex V-100 in two; Contour in two; Biotronik Phylax 06 in one; and Telectronic Guardian ATP2/3 in one.

\section{Postoperative period}

Five patients died within the first 30 days after implantation. In one patient with congenital heart disease and multiple previous surgical procedures, perforation of the subclavian vein led to haemothorax which, in conjunction with advanced liver 


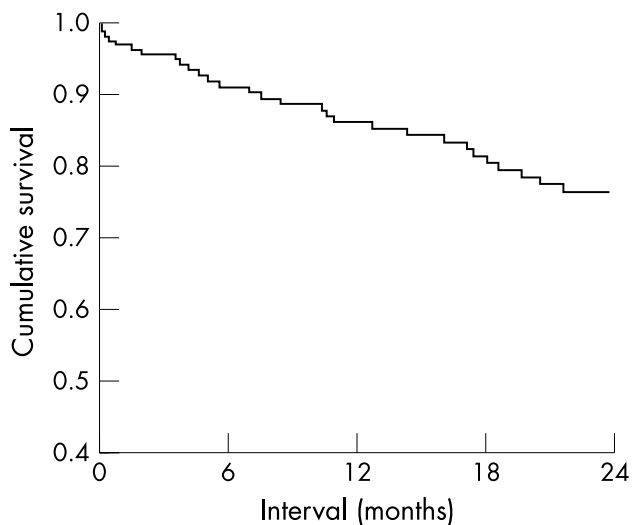

Figure 1 Total mortality in 165 patients with New York Heart Association (NYHA) functional class III presenting either with ventricular tachycardia or ventricular fibrillation.

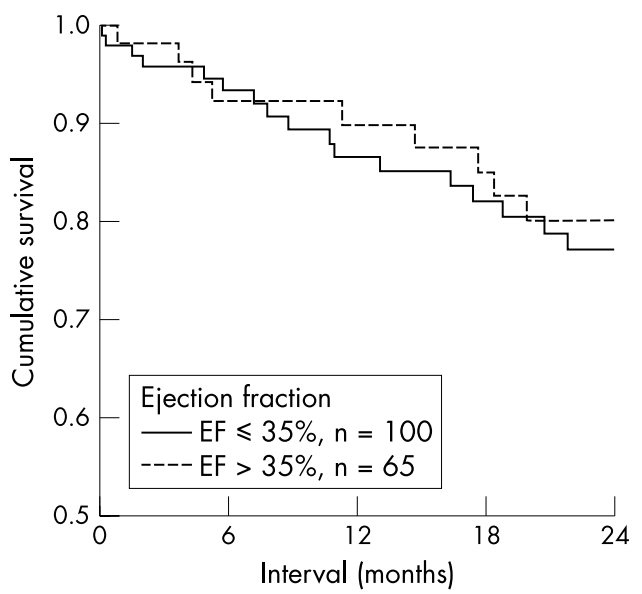

Figure 2 Cardiac mortality and ejection fraction (EF). Outcome was similar between subgroups ( $\mathrm{EF} \leqslant 35 \%$ and $\mathrm{EF}>35 \%$ ).

disease, caused the patient's death five days after implantation. Two patients died because of heart failure on day 3 and day 24 after implantation. Another patient died because of resistant incessant VT and heart failure on day 6 after implantation. One further patient died from pulmonary embolism 12 days after implantation of an epicardial lead system. The mean ejection fraction in the patients who died perioperatively was $36(8) \%$.

\section{Long term outcome}

During a follow up of 23 (20) months, 40 deaths occurred, most within the first two years (fig 1). Thirty four of these were classified as cardiac, 11 of which were sudden. The incidence of cardiac death was $9.6 \%$ and $15.1 \%$ at 12 and 24 months, respectively. The sudden death incidence (not shown) was $2.4 \%$ and $6.3 \%$ at 12 and 24 months. Death from any other

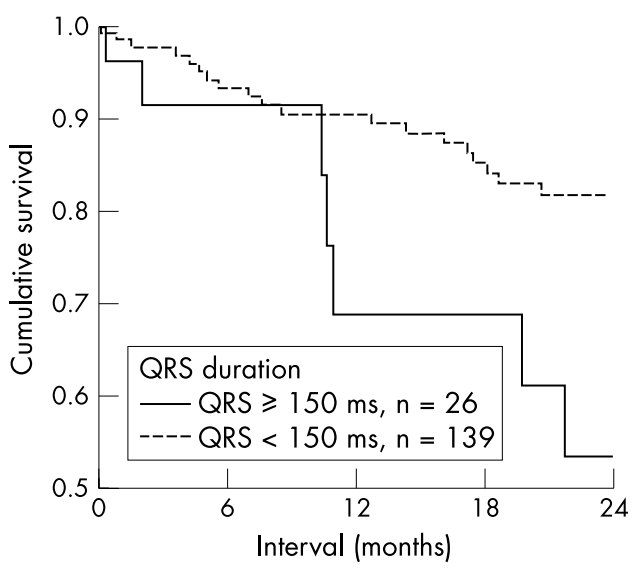

Figure 3 Cardiac mortality and QRS duration. Patients with a prolonged QRS duration ( $\geqslant 150 \mathrm{~ms}$ ) had a significantly worse outcome than patients with a QRS duration of $<150 \mathrm{~ms}$.

cause occurred in $2.0 \%$ after 12 and 24 months. The overall mortality was $14 \%$ and $23 \%$ at 12 and 24 months. VT recurred in $59 \%$ and $70 \%$ within 12 and 24 months. Fast VT $(\geqslant 240$ beats/min), which would presumably have been lethal without termination by the device, occurred in $22 \%$ and $28 \%$ within 12 and 24 months.

\section{Ejection fraction and mortality and recurrences of VT or fast VT}

The survival rates at 12 and 24 months did not differ in patients with EF > 35\% compared with those with EF $\leqslant 35 \%$ (fig 2). Cardiac mortality, sudden death rates, or recurrence rates of any ventricular tachyarrhythmia were similar in the two groups (table 2 ). The risk ratio for cardiac mortality using a cut off of $35 \%$ was 1.235 (95\% confidence interval (CI) 0.609 to 2.504$)$. The recurrence of fast VT ( $\geqslant 240$ beats/min) was similar in the group with EF $\leqslant 35 \%$ and $>35 \%$ (77\% v $68 \%$ at 24 months, NS).

\section{QRS duration and mortality}

At the end of follow up, overall mortality from any cause was similar in patients with a QRS duration of $<150$ ms (group l, $52.4 \%$ ) and in those with a QRS duration of $\geqslant 150 \mathrm{~ms}$ (group $2,46.6 \%)$, as evaluated by Kaplan-Meier analysis $(\mathrm{p}=0.13)$. However, cardiac mortality was significantly higher in patients with an increased QRS duration (group 2). Kaplan-Meier analysis showed that cardiac mortality in group 1 at 12 and 24 months was $9.5 \%$ and $18.2 \%$, respectively, compared with $31.3 \%$ and $46.6 \%$ in group 2 (increased QRS duration) $(p=0.04)$ (fig 3$)$. Thus in patients with heart failure, increased cardiac mortality was associated with a widened QRS (risk ratio 2.178 , 95\% CI 0.946 to 5.055 ). This effect appeared to have prognostic importance, especially in the long term. The difference in mortality between the two groups increased in the second year of follow up (fig 3).

Table 2 Survival according to ejection fraction

\begin{tabular}{llllll}
\hline \multirow{2}{*}{ Survival/event-free interval } & \multicolumn{2}{l}{ EF $>35 \%$} & & \multicolumn{2}{l}{ EF $\leqslant 35 \%$} \\
\cline { 2 - 3 } \cline { 5 - 5 } Overall mortality & 6 months & 24 months & & 6 months & 24 months \\
\hline Cardiac death & $87.4 \%$ & $75.9 \%$ & $93.3 \%$ & $77.0 \%$ \\
Sudden death & $92.2 \%$ & $80.0 \%$ & $93.3 \%$ & $77.0 \%$ \\
VT & $97.8 \%$ & $89.7 \%$ & & $100 \%$ & $94.7 \%$ \\
Fast VT & $58.9 \%$ & $34.7 \%$ & & $57.3 \%$ & $26.5 \%$ \\
\cline { 1 - 2 } & $85.0 \%$ & $76.9 \%$ & $85.3 \%$ & $67.7 \%$ \\
\hline
\end{tabular}

Actuarial survival rates and intervals free of recurrence of ventricular tachycardia in relation to ejection fraction (cut off value 35\%). There were no significant differences between subgroups.

$E F$, ejection fraction; fast VT, ventricular tachycardia of $\geqslant 240$ beats/min; VT, ventricular tachycardia. 


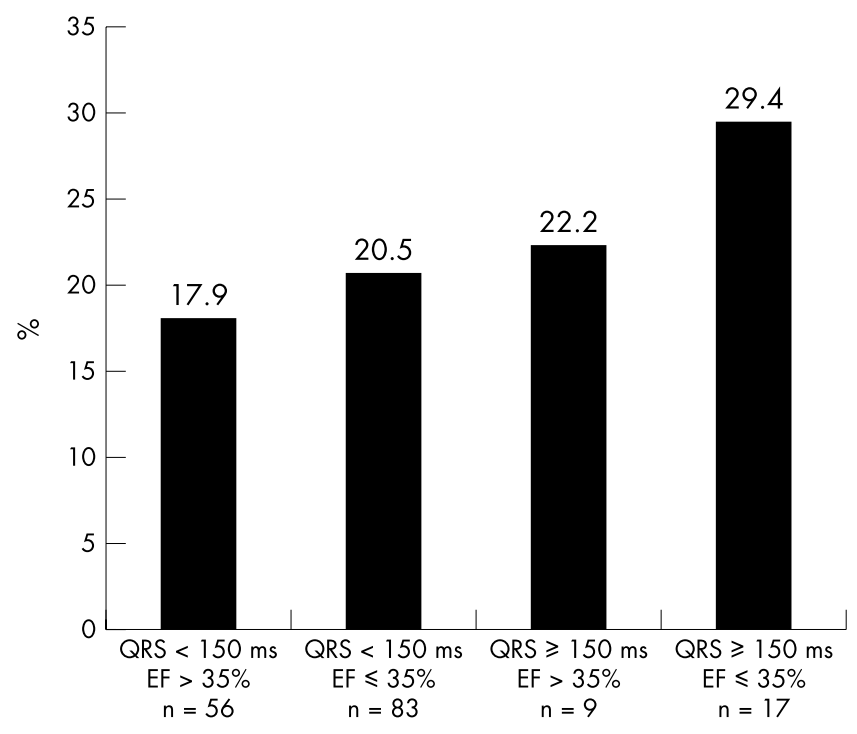

Figure 4 Cardiac mortality and QRS duration. In the absence of an increased QRS duration and with relatively well preserved left ventricular function (ejection fraction (EF) $>35 \%$ ), cardiac mortality rate was $17.9 \%$. In contrast, if the QRS duration was $\geqslant 150 \mathrm{~ms}$ and $\mathrm{EF} \leqslant 35 \%$, cardiac mortality rose to $29.4 \%$.

\section{QRS duration and recurrence of VT}

Event-free intervals for VT recurrence did not differ between group 1 and group 2. However, patients with an increased QRS duration (group 2) had more recurrences of fast VT $(50 \%, v$ $26 \%$ in group 1 after 24 months; $p=0.03)$.

\section{QRS duration and left ventricular ejection fraction: predictors of cardiac mortality}

As outlined in fig 4, in the presence of a depressed left ventricular ejection fraction ( $\leqslant 35 \%)$ and an increased QRS duration, overall cardiac mortality was $29.4 \%$ (over the whole period of follow up). If neither variable was present, overall mortality was $17.9 \%$. Thus an increased QRS duration appeared to contribute to the risk of cardiac death in patients with heart failure fitted with an ICD.

\section{DISCUSSION}

Our principal finding was that in this group of patients with NYHA grade III heart failure, those with an increased QRS duration had a greater cardiac mortality. It is not uncommon for patients with advanced heart failure to develop significant interventricular and intraventricular conduction delays. ${ }^{8-14} 18$ Several studies have suggested that a prolonged QRS duration is an independent or contributing risk factor for mortality in patients with heart failure. Shamim and colleagues studied the outcome of 172 patients with heart failure. ${ }^{10}$ Mortality in the 50 patients with a QRS duration of $\geqslant 140 \mathrm{~ms}$ was $50 \%$, as opposed to $23 \%$ in the remaining patients. In patients with severe heart failure awaiting transplantation, Aaronson and colleagues developed multivariable proportional hazard survival models on 80 clinical characteristics of patients with heart failure. ${ }^{11}$ Invasive and non-invasive models were constructed. The non-invasive model performed well in discriminating one year event-free survival in high versus low risk groups. An intraventricular conduction delay predicted mortality with an adjusted hazard ratio of 1.84 (95\% CI 1.22 to $2.76 ; \mathrm{p}=0.0035)$. Xiao and colleagues studied 58 patients with dilated cardiomyopathy. ${ }^{12}$ Patients with a QRS duration of $\geqslant 160 \mathrm{~ms}(\mathrm{n}=10)$ had a mortality of $80 \%$, compared with $13 \%$ in the remaining patients. In an early study of 33 patients with congestive cardiomyopathy, there was a tendency for the QRS duration to increase during the course of the disease. ${ }^{18}$ Senni and colleagues reported the clinical characteristics and natural history in 216 patients with their first diagnosis of heart failure in a single US county in $1991^{19} ; 88 \%$ were older than 65 years and 54\% were in NYHA grade III or IV. Fifty patients $(23 \%)$ had either left bundle branch block or an intraventricular conduction delay which was predictive of a left ventricular ejection fraction of less than $50 \%$. Overall survival was $86 \%$ at three months, $76 \%$ at one year, and $35 \%$ at five years.

In the present study in patients with NYHA grade III heart failure and documented VT or VF, the prevalence of conduction abnormalities was 16\% (26 of 165 patients). Stellbrink and colleagues retrospectively analysed data from 384 patients for NYHA class, QRS duration, and incidence of atrial fibrillation at the time of ICD implantation. ${ }^{20}$ Patients with advanced congestive heart failure were older and had an increased QRS duration; 53\% of patients with NYHA grade III heart failure had a QRS duration of $\geqslant 120 \mathrm{~ms}$, and 37\% (48 of 129 patients) had a QRS width of $\geqslant 150 \mathrm{~ms}$ - somewhat higher than the $16 \%$ in the present study.

Possible clinical consequences of ventricular asynchrony include abnormal interventricular septal wall motion, a reduction in the peak rate of rise of left ventricular pressure, reduced diastolic filling times, and prolonged duration of mitral regurgitation. ${ }^{8}$ Within the subgroup of patients with a depressed left ventricular ejection fraction $(\leqslant 35 \%)$, the presence of an increased QRS duration was associated with a greater cardiac mortality. Several studies that have included signal averaging methods have shown that an increased QRS duration of the filtered QRS complex identifies postmyocardial infarction patients at risk of arrhythmic deaths. ${ }^{21-23}$ In addition, the GUSTO-I investigators analysed the initial ECG at the time of acute infarction to predict all cause mortality. ${ }^{24}$ An increased QRS duration in the presence of acute anterior infarction was predictive of mortality (odds ratio 1.55). In the cardiac arrhythmia suppression trial (CAST), Josephson and colleagues recently analysed multiple variables to predict mortality of patients with poor left ventricular function. ${ }^{4}$ Along with previous coronary bypass grafting, basic heart rate, and diastolic blood pressure, QRS duration was a strong and simple predictor of mortality. The prognostic value of QRS width-a simple, non-invasive, and reliable index in ICD recipients-has not yet been analysed. Thus, independently of NYHA functional status and impairment of left ventricular function, QRS width is a marker of increased cardiac mortality in ICD recipients. This has not been reported previously, despite assessments of the effects of variables predicting outcome in ICD recipients.

Sheldon and colleagues recently undertook a retrospective analysis of the Canadian implantable defibrillator study (CIDS) to predict those patients who might benefit most from an ICD. ${ }^{15}$ In the group at highest risk, there was a $50 \%$ relative reduction of mortality in patients fitted with ICDs. Patients who were most likely to benefit from an ICD could be identified by a simple risk score (two or more of the following risk factors): age $\geqslant 70$ years, left ventricular ejection fraction $\leqslant 35 \%$, and NYHA class III or IV. Thirteen of 15 deaths prevented by the ICD occurred in patients with two or more risk factors. The implications of these findings regarding the clinical selection of patients for ICD and the cost effectiveness of using expensive ICD therapy primarily in the group of patients at highest risk are self evident. The findings from the CIDS group are in agreement with a recent paper by Domanski and colleagues from the AVID investigators. ${ }^{25}$ The AVID data indicate that patients with a relatively well preserved ejection fraction $(\geqslant 35 \%)$ do not have better survival when treated with the ICD than with antiarrhythmic drugs. However, in patients with a lower ejection fraction, the ICD was associated with improved survival. Relatively improved survival occurred in patients with an ejection fraction in the range of $20-34 \%$, as well as in those with a value of less than $20 \%$, although in the latter group this difference was non-significant. 
In MADIT (multicenter automatic defibrillator implantation trial), Moss reported an increased benefit of the ICD over conventional medical treatment in the subgroup of patients with an EF $<26 \%$, as opposed to those with a value between $26-35 \% .{ }^{26}$ The eligibility criteria for MADIT were an ejection fraction of $\leqslant 35 \%$ and non-sustained VT on Holter monitoring (primary prevention study). ${ }^{27}$ It should be mentioned that in MADIT, as in our study, there was a disparity in $\beta$ blocker use between the subgroups. Böcker and colleagues also showed a substantial benefit of ICD treatment in patients with more advanced heart failure, using hypothetical event calculations based on recurrence of potentially lethal VT/VF (fast VT $\geqslant 240$ beats/min). ${ }^{16}$ Thus patients with moderate to severe left ventricular dysfunction obtained the greatest benefit from ICD treatment.

Although mortality generally increases with increasing NYHA functional class, the proportion of deaths that are sudden declines from $50-80 \%$ in class II to $30 \%$ in class IV. ${ }^{28}$ The sicker patients are more likely to die from progressive heart failure than suddenly from fatal bradyarrhythmias or electromechanical dissociation. ${ }^{29}$ As the various mechanisms of death have different frequencies depending on the severity of the heart failure, the prediction of arrhythmic deaths in such patients is difficult. In the present study, an increased QRS duration identified patients with increased cardiac mortality in the presence of depressed left ventricular function. This finding has implications for the selection of patients who will benefit from an ICD over time; for stratifying those patients eligible for heart transplantation at the time of implantation if no further contraindications exist; and for implementation of biventricular pacing devices combined with ICD treatment in patients with heart failure.

Recent preliminary acute and short term studies employing pacing (either biventricular or left ventricular) have shown a significant clinical improvement in patients with heart failure. ${ }^{30-33}$ Although the long term outcome of pacing therapy in heart failure is presently being assessed in prospective controlled studies, the data from our analysis indicate that the influence of biventricular pacing on mortality is probably best studied in patients with heart failure and documented VT or $\mathrm{VF}$, as the overall event rates in patients with an increased QRS duration are high. ${ }^{17}$ Thus relatively small numbers of patients would need to be included in a prospective trial to gain a better understanding of the role of biventricular pacing in improving the prognosis in patients with heart failure.

\section{Limitations}

The total number of patients available for analysis was small in the light of recently published large prospective defibrillator trials. However, even with these limited numbers of patients, statistically meaningful outcome data could be provided.

Only $17 \%$ of the patients included in the analysis had dilated cardiomyopathy and the majority of cases (73\%) had coronary artery disease. As yet, we do not know whether these data are applicable to patients with dilated cardiomyopathy.

Dynamic changes of QRS duration over time were not analysed in the present study. The absence of serial measurements (progression of disease) limits the clinical applicability.

Medical treatment with $\beta$ blockers (26\%) and angiotensin converting enzyme inhibitors (73\%) was being given at the time of ICD implantation. Although there were no significant differences in drug treatment on discharge within the two subgroups, the low $\beta$ blocker use in this heart failure cohort may be a cause for concern.

\section{Conclusions}

Our study suggests that an increased QRS duration is a positive predictor of cardiac death in patients with NYHA functional class III heart failure and documented VT or VF. This simple, non-invasive, and easily measured index may help to determine the cost effectiveness of ICDs in the secondary prevention of sudden and cardiac death in patients with heart failure.

\section{ACKNOWLEDGEMENT}

This study was in part supported by the Franz Loogen Foundation, Düsseldorf.

\section{Authors' affiliations}

L Bode-Schnurbus, D Böcker, M Block, R Gradaus, G Breithardt, Department of Cardiology and Angiology and Institute for Research in Arteriosclerosis, Westfälische Wilhelms-University, Münster, Germany A Heinecke, Institute for Biomathematics, Münster, Germany M Borggrefe, Department of Internal Medicine, University Hospital, Mannheim, Germany

\section{REFERENCES}

1 Smith WM. Epidemiology of congestive heart failure. Am J Cardiol 1985;55:3-8A.

2 Schocken DD, Arrieta MI, Leaverton PE, et al. Prevalence and mortality rate of congestive heart failure in the United States. J Am Coll Cardiol 1992;20:301-6.

3 Singh SN, Fletcher RD, Fisher SG, et al. Amiodarone in patients with congestive heart failure and asymptomatic ventricular arrhythmia. N Engl J Med 1995;333:77-82.

4 Josephson RA, Chahine RA, Morganroth J, et al. Prediction of cardiac death in patients with a very low ejection fraction after myocardial infarction: a cardiac arrhythmia suppression trial (CAST) study. Am Heart J 1995;130:685-91.

5 The AVID Investigators. A comparison of antiarrhythmic drug therapy with implantable defibrillators in patient resuscitated from near-fatal ventricular arrhythmias. N Engl J Med 1997;337:1576-83.

6 Conolly SJ, Gent M, Roberts RS, et al. Canadian implantable defibrillator study (CIDS): a randomized trial if the implantable cardioverter defibrillator against amiodarone. Circulation 2000;101:1297-302.

7 Kuck KH, Cappato R, Siebels J, et al. A randomized comparison of antiarrhythmic drug therapy with implantable defibrillators in patients resuscitated from cardiac arrest. Circulation 2000;102:748-54.

8 Grines CL, Bashore TM, Boudoulas H, et al. Functional abnormalities in isolated left bundle branch block. The effect of intraventricular asynchrony. Circulation 1989;79:845-53.

9 Xiao HB, Brecker JD, Gibson DG. Effect of abnormal activation on the time course of the left ventricular pressure pulse in dilated cardiomyopathy. Br Heart J 1992;68:403-7.

10 Shamim W, Francis DP, Yousufuddin $M$, et al. Intraventricular conduction delay. A predictor of mortality in chronic heart failure? [abstract] Eur Heart J 1998;19:a926.

11 Aaronson KD, Schwartz JS, Chen TM, et al. Development and prospective validation of a clinical index to predict survival in ambulatory patients referred for cardiac transplant evaluation. Circulation 1997;95:2660-7.

12 Xiao HB, Roy C, Fujimoto S, et al. Natural history of abnormal conduction and its relation to prognosis in patients with dilated cardiomyopathy. Int J Cardiol 1996;53:163-70.

13 Schoeller $\mathbf{R}$, Andresen D, Büttner $\mathrm{P}$, et al. First- or second-degree atrioventricular block as a risk factor in idiopathic dilated cardiomyopathy. Am J Cardiol 1993;71:720-6.

14 Wilensky RL, Yudelman P, Cohen Al, et al. Serial electrocardiographic changes in idiopathic dilated cardiomyopathy confirmed at necropsy. Am J Cardiol 1988;62:276-83.

15 Sheldon R, Conolly S, Krahn A, et al. Identification of patients most likely to benefit from implantable cardioverter-defibrillator therapy. The Canadian implantable defibrillator study (CIDS). Circulation 2000;101:1660-4.

16 Böcker D, Bänsch D, Heinecke A, et al. Potential benefit from implantable cardioverter-defibrillator therapy in patients with and without heart failure. Circulation 1998;98:1636-43.

17 Gras D, Ritter P, Lazarus A, et al. Long-term outcome of advanced heart failure patients with cardiac resynchronization therapy. Pacing Clin Electrophysiol 2000;23:658.

18 Kuhn H, Breithard LK, Breithardt G, et al. Die Bedeutung des Elektrokardiogramms für die Diagnose und Verlaufsbeobachtung von Patienten mit kongestiver Kardiomyopathie. Z Kardiol 1974;63:916-27.

19 Senni M, Tribouilloy CM, Rodeheffer RJ, et al. Congestive heart failure in the community: a study of all incident cases in Olmsted County, Minnesota, in 1991. Circulation 1998;98:2282-9.

20 Stellbrink C, Auricchio A, Diem B, et al. Potential benefit of biventricular pacing in patients with congestive heart failure and ventricular tachyarrhythmia. Am J Cardiol 1999;83:143-50D.

21 Hartikainen JE, Malik M, Staunton A, et al. Distinction between arrhythmic and nonarrhythmic death after acute myocardial infarction based on heart rate variability, signal-averaged electrocardiogram, ventricular arrhythmias and left ventricular ejection fraction. J Am Coll Cardiol 1996;28:296-304. 
22 El-Sherif N, Denes P, Katz R, et al. Definition of the best prediction criteria of the time domain signal-averaged electrocardiogram for serious arrhythmic events in the postinfarction period. The cardiac arrhythmia suppression trial/signal averaged electrocardiogram (CAST/SAECG) Substudy Investigators. J Am Coll Cardiol 1995;25:908-14.

23 Reinhardt L, Makijarvi M, Fetsch T, et al. Noninvasive risk modeling after myocardial infarction. Am J Cardiol 1996;78:627-32.

24 Hathaway WR, Peterson ED, Wagner GS, et al. Prognostic significance of the initial electrocardiogram in patients with acute myocardial infarction. GUSTO-I Investigators. Global utilization of streptokinase and t-PA for occluded coronary arteries. JAMA 1998;279:387-91.

25 Domanski MJ, Sakseena S, Epstein AE, et al. (for the AVID Investigators.) Relative effectiveness of the implantable cardioverter-defibrillator and antiarrhythmic drugs in patients with varying degrees of left ventricular dysfunction who have survived malignant ventricular arrhythmias. J Am Coll Cardiol 1999;34:1090-5.

26 Moss AJ. Implantable cardioverter defibrillator therapy. The sickest patients benefit the most. Circulation 2000;101:1638-40.

27 Moss AJ, Hall WJ, Cannom DS, et al. (for the Multicenter Automatic Defibrillator Implantation Trial Investigators.) Improved survival with an implanted cardioverter defibrillator in patients with coronary artery disease at high risk for ventricular arrhythmia. N Engl J Med 1996;335: 1933-40.

28 Uretsky BF, Sheahan RG. Primary prevention of sudden cardiac death in heart failure: will the solution be shocking? J Am Coll Cardiol 1997:30: 1589-97.

29 Stevenson WG, Stevenson LW, Middlekauff HR, et al. Sudden death prevention in patients with advanced ventricular dysfunction. Circulation 1993;88:2953-61

30 Leclercq C, Cazeau S, Le Breton $\mathrm{H}$, et al. Acute hemodynamic effects of biventricular DDD pacing in patients with end-stage heart failure. J Am Coll Cardiol 1998;32:1825-31.

31 Kass DA, Chen $\mathrm{CH}$, Curry C, et al. Improved left ventricular mechanics from acute VDD pacing in patients with dilated cardiomyopathy and ventricular conduction delay. Circulation 1999;99:1567-73.

32 Auricchio A, Stellbrink C, Block M, et al. Effect of pacing chamber and atrioventricular delay on acute systolic function of paced patients with congestive heart failure. Circulation 1999:99:2993-3001.

33 Blanc JJ, Etienne $Y$, Gilard $M$, et al. Evaluation of different ventricular pacing sites in patients with severe heart failure: results of an acute hemodynamic study. Circulation 1997;96:3273-7.

\section{IMAGES IN CARDIOLOGY}

\section{$\beta$ Thalassaemia major and severe left ventricular dysfunction}

$\mathrm{P}$ atients with $\beta$ thalassaemia major are dependent upon regular blood transfusions, and consequently are subjected to a large iron load. Accumulation of excess iron leads to heart failure. Iron chelation therapy can delay this process, but compliance is commonly poor. Severe cardiac failure in iron overloaded patients with $\beta$ thalassaemia major has previously been reported as being invariably fatal.

A 21 year old man with $\beta$ thalassaemia major was admitted with breathlessness. Examination revealed tachycardia, hypotension, and tachypnoea. He had a third heart sound, ankle oedema, raised venous pressure, smooth hepatomegaly, and dullness at the right base. Chest $x$ ray showed cardiomegaly and a right pleural effusion. Ferritin was notably elevated $(>5000 \mu \mathrm{g} / \mathrm{l}$ ). ECG showed sinus tachycardia. An echocardiogram (below left) showed severe left ventricular impairment (ejection fraction $<20 \%$ ).

Despite diuretics the patient continued to decline. Inotrope and high dose desferroxiamine $(70 \mathrm{mg} / \mathrm{kg} /$ day) intravenous infusions were initiated. He showed clinical improvement, inotropes were withdrawn after nine days, and he was

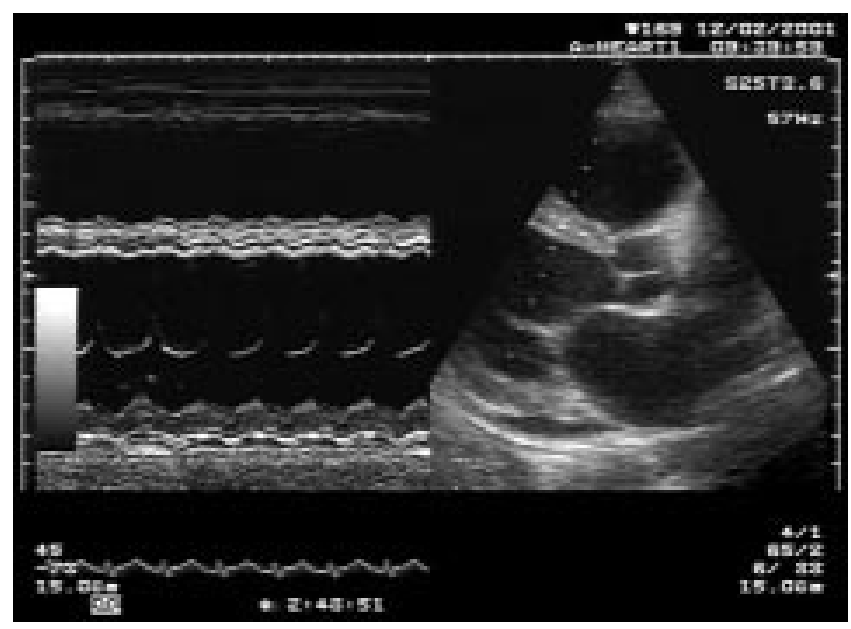

eventually discharged home on oral frusemide (furosemide), spironolactone, ramipril, and chelation therapy (subcutaneous desferrioxamine and oral deferiprone).

Four months post-presentation he underwent cardiac T2* magnetic resonance imaging, which showed severe iron overload (cardiac T2* $9.1 \mathrm{~ms}$; normal range $20-83 \mathrm{~ms}$ ). After three months his cardiac T2* improved marginally $(9.7 \mathrm{~ms})$, and his ejection improved to within the normal range $(57 \%$, below right).

This case demonstrates that patients with severe cardiac failure caused by transfusional iron overload should receive full treatment including inotropic support since recovery is possible. High dose chelation therapy probably contributes to this improvement.

M D Sosin

S Handa

D L Connolly

R Davis

r.davis@swbh.nhs.uk

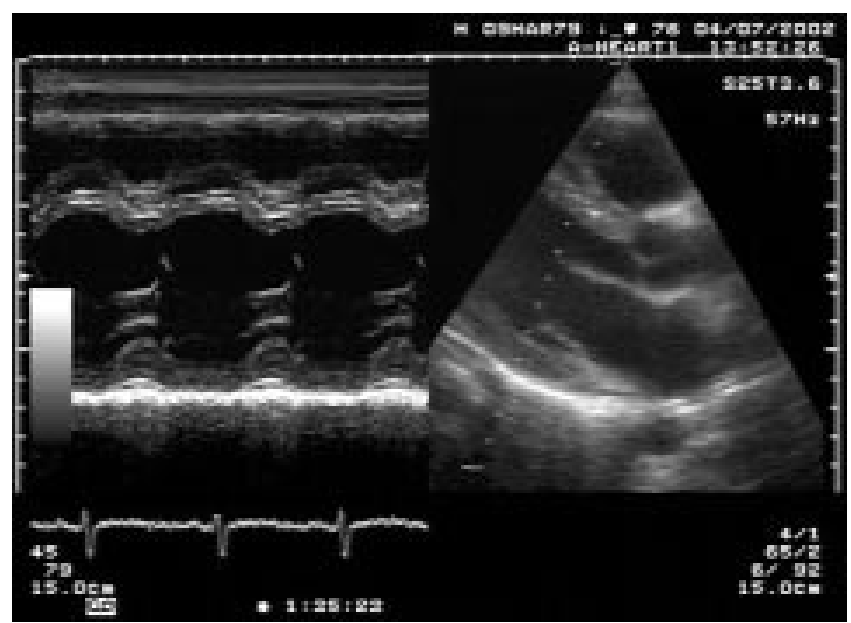

